\title{
The use of urinary bladder matrix in the treatment of trauma and combat casualty wound care
}

Treatment of combat injuries and resulting wounds can be difficult to treat due to compromised and evolving tissue necrosis, environmental contaminants, multidrug resistant microbacterial and/or fungal infections, coupled with microvascular damage and/or hypovascularized exposed vital structures. Our group has developed surgical care algorithms with identifiable salvage techniques to achieve stable, definitive wound coverage often with the aid of certain regenerative medicine biologic scaffold materials and advanced wound care to facilitate tissue coverage and healing. This case series reports on the role of urinary bladder matrix scaffolds in the wound care and reconstruction of traumatic and combat wounds. Urinary bladder matrix was found to facilitate definitive soft tissue reconstruction by establishing a neovascularized soft tissue base acceptable for second stage wound and skin coverage options within traumatic and combat-related wounds.

Keywords: biologic scaffolds $\bullet$ combat casualty care $\bullet$ extracellular matrix $\bullet$ extremity trauma - limb salvage $\bullet$ regenerative medicine $\bullet$ soft tissue regenerate $\bullet$ urinary bladder matrix

- vascularized wound bed

The military engagements in Operations Iraqi Freedom and Enduring Freedom (OIF/OEF) have been characterized by wounds caused predominantly by ballistic and blast mechanisms of injury [1-11]. These wounds consist of massive soft tissue and orthopedic injuries secondary to the following mechanisms: damage due to pressure waves and forces exerted on bodily tissues, penetrating trauma, contamination with environmental particulates, burnrelated tissue destruction and blunt trauma from being 'thrown' by the explosion force [12]. Combat casualty survivors often have high injury severity scores with increasing rates of concomitant injuries and multiorgan system involvement [10]. Their associated extremity wounds may exhibit major neurovascular disruptions, exposed orthopedic fracture(s) and tendinous injuries devoid of periosteum and/or paratenon, coupled with massive soft tissue volumetric loss. These critical injuries may be in continuity or disparate and tend to worsen with time due to tissue ischemia over the entire zone of injury and increased infection rates from the direct inoculation of particulate matter.

Throughout the care of our trauma patients, serial surgical debridement procedures are required to gain control of such wounds prior to advanced reconstruction and/or implementation of regenerative modalities to gain definitive wound coverage [10]. The ultimate goals of these definitive reconstruction procedures are to: achieve durable wound and/or skin coverage to recapitulate the barrier and protective roles of skin; preserve the functional capacity of extremities and residual limbs that have been affected by complex wounding mechanisms; preserve as much soft tissue, nerve, tendon, and bone as possible; and achieve stable long-term coverage, and closure of these wounds [8-10].

The magnitude and complexity of traumatic and blast wounds may require utilizing multiple techniques from the traditional reconstructive ladder/elevator, which may
Ian L Valerio*,1,2,3, Paul Campbell ${ }^{4}$, Jennifer Sabino ${ }^{4}$, Christopher L Dearth ${ }^{5,6}$ \& Mark Fleming 3,7 'Department of Plastic \& Reconstructive Surgery, Division of Burn, Wound \& Trauma, Wexner Medical Center of the Ohio State University, 915 Olentangy River Road, Ste 2100, Columbus, OH, USA

2Plastic \& Reconstructive Surgery Service, Department of Surgery, Walter Reed National Military Medical Center, Bethesda, MD, USA

${ }^{3}$ Department of Surgery, Uniformed

Services University of the Health

Sciences, Bethesda, MD, USA

${ }^{4}$ Department of Surgery, Walter Reed National Military Medical Center, Bethesda, MD, USA

${ }^{5}$ DoD-VA Extremity Trauma

\& Amputation Center of Excellence, Walter Reed National Military Medical Center, Bethesda, MD, USA

${ }^{6}$ Department of Physical Medicine \& Rehabilitation, Uniformed Service University of the Health Sciences, Bethesda, MD, USA

${ }^{7}$ Department of Orthopedics, Walter Reed National Military Medical Center, Bethesda, MD, USA

*Author for correspondence:

Tel.: +1 4127283377

iv_cwru@yahoo.com 
often be augmented with regenerative medicine and biologic wound care therapies in an effort to provide stable, definitive soft tissue coverage (Figure 1). It is not uncommon for a single combat casualty to undergo local wound care with negative pressure therapies, extracellular matrices (ECM) placement, secondary skin grafting, local/pedicle flaps and free tissue transfers (free flaps). Thus, military reconstructive teams have explored the use of novel regenerative modalities earlier within the treatment course of trauma care in the goal of successfully achieving definitive wound coverage and healing [10].

Traditionally, ECMs were thought to merely provide structural support for tissues; however it is now widely recognized that ECMs may have much more biologic significance. ECM scaffold materials are derived from the decellularization of many tissue sources (e.g., urinary bladder $[\mathrm{UBM}]$, small intestinal submucosa [SIS], dermis [D-ECM]) that arise from a number of different species (e.g., human, porcine, bovine, ovine, shark) [13-20]. ECM scaffolds consist of a tissue source specific three-dimensional arrangement of structural and functional molecules such as collagen, laminin, fibronectin, glycosaminoglycans and growth factors.
This milieu of biochemical and structural signals provides a microenvironmental niche ideally suited for mediating constructive tissue remodeling that can aid in more effective wound healing.

A variety of naturally occurring scaffold materials composed of ECM have been used to support regeneration/remodeling, rather than the default fibrotic scarring, of various tissue types after injury. Importantly, the new host tissue generated in the proximity of ECM scaffold materials may be similar to, but does not perfectly recapitulate, the native tissue structure and function. The exact mechanisms of ECM scaffold mediated constructive remodeling require further elucidation. However, it is currently known that within hours of being surgically placed at a site of tissue injury, ECM scaffolds are infiltrated by host derived mononuclear cells, which facilitate proteolytic degradation of the scaffold thereby generating a variety of bioactive molecules including matricryptic peptides [19,20]. These events are important in orchestrating the complex host response that ensues - antibacterial and angiogenic activity, the migration, proliferation, and/or differentiation of endogenous multipotent progenitor cells, and modulation

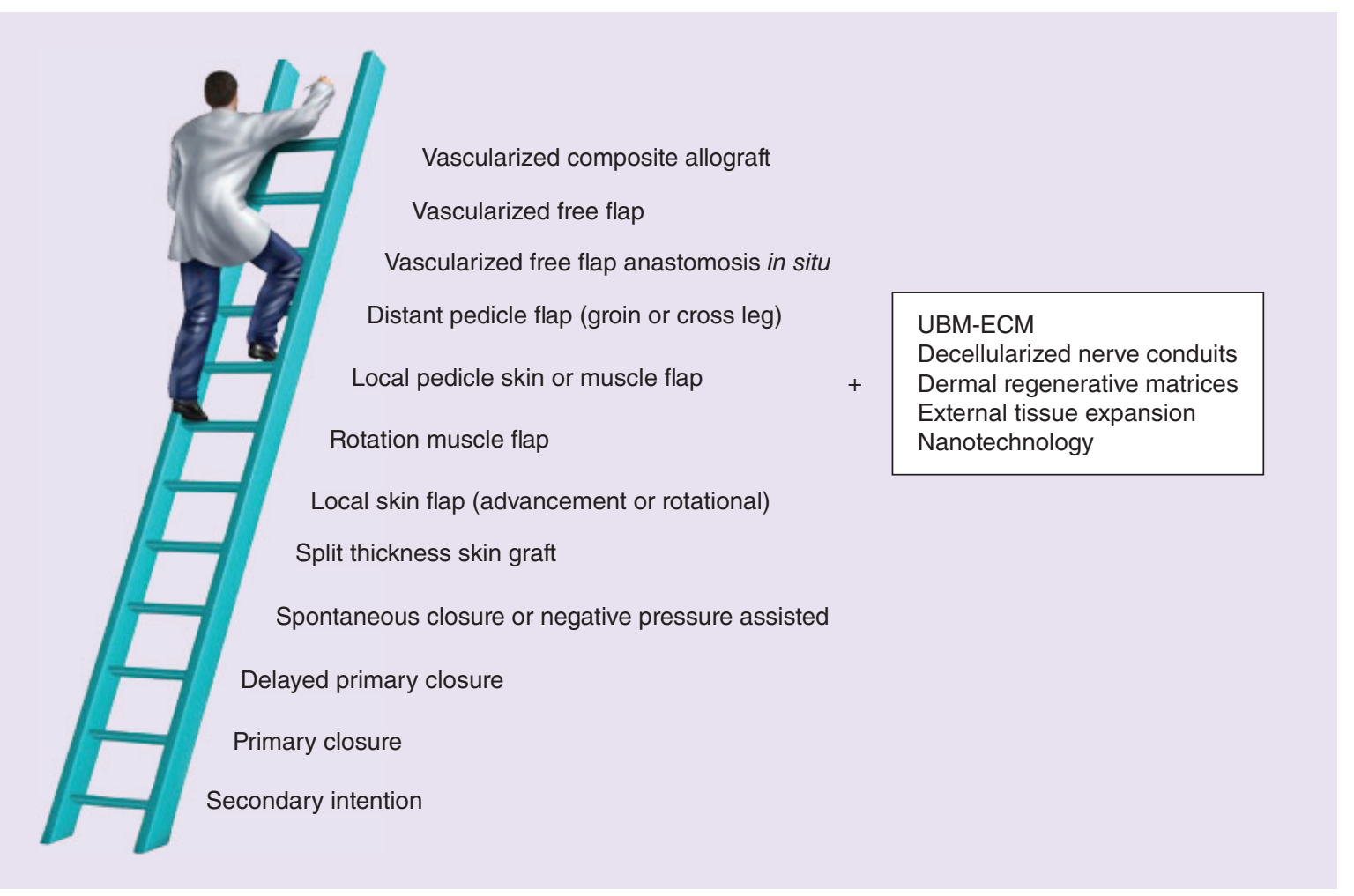

Figure 1. Example of the hybrid reconstructive ladder. The reconstruction ladder describes increasingly complex soft tissue coverage techniques to achieve wound coverage. Traditional reconstruction techniques can be augmented with regenerative medicine modalities, which may facilitate staying on a lower 'rung' to achieve wound coverage (Reproduced with permission from www.123rf.com - an open access site with license granted to publish).

UBM-ECM: Urinary bladder matrix-extracellular matrix. 
of the local immune response toward potentially anti-inflammatory macrophage phenotype [13,21-25].

Given the favorable remodeling responses facilitated by ECM scaffold materials, it is plausible that reconstruction of certain combat wounds would benefit from their use in order to obtain viable vascularized wound beds and the establishment of healthy granulation tissue. This clinical case series reports the findings of a retrospective review of traumatic and combat casualty cases at Walter Reed National Military Medical Center which utilized UBM in the wound care and reconstruction of various traumatic and combat related wounds.

\section{Materials \& methods}

Traumatic and combat wound care cases utilizing UBM at Walter Reed National Military Medical Center from 2010 through 2014 were reviewed after appropriate clearances and institutional approved consent process including medical and surgical photograph use permissions. UBM is an acellular, noncrosslinked, resorbable ECM biologic scaffold, available in powder (MatriStem MicroMatrix ${ }^{\circledR}$, ACell, Inc., MD, USA) and sheet forms (MatriStem Burn and Wound Matrices, ACell, Inc.). Preoperative and serial photographs were taken of each patient's wounds. Wound care therapies and reconstruction techniques that were used during the care of these patients were reviewed. Overall tissue remodeling outcomes including complications such as wound care and/or reconstructive failures, infections (cellulitis or abscess formation), partial or complete loss of ECMs, UBMs, grafts and/or flaps were recorded.

All wounds were treated according to the standards of care at our organization, with serial irrigation and debridement procedures to remove particulate matter and necrotic or infected tissue occurring prior to considering reconstruction. Negative pressure wound therapy (NPWT) was administered often at the initial in-theater military treatment facilities and maintained during the course of serial wound treatment at our center. Serial surgical irrigation and debridement procedures were performed every 2-3 days until the patient was medically optimized and their wounds were considered ready for definitive reconstruction measures.

Wound care was approached by a multidisciplinary team with general, orthopedic and plastic and reconstructive surgery personnel participating in management. UBM was used at the discretion of the operating surgeon. UBM materials consisted of either a powder and/or sheet form and were used separately or in combination at the surgeon's discretion. The timing of application, amount of product used and wound care regimen were also at the surgeon's discretion and based on the injury pattern and needs of the individual patients.

Typically, the preferred surgical technique involved surgically preparing the wound bed with debridement of nonviable tissue and irrigation with copious amounts of sterile saline (minimal of 31 or more depending on the size of the wound). After completing the aforementioned irrigation and debridement, wounds with exposed structures devoid of adequate vascularity (e.g., tendons devoid of paratenon, bone devoid of periosteum and among others) received an application of UBM powder $(50 \mathrm{mg}$ up to $1000 \mathrm{mg}$ ) to completely cover the exposed poorly vascularized vital structure with a thin visible layer of powder. The powder was then covered with a lyophilized UBM burn or wound sheet that was pre-moistened with sterile saline. To prevent the escape of the underlying and covered powder, the sheet was secured into place with 4-0 chromic suture. This technique was repeated every 3 days if the structures still appeared to lack an acceptable bed suitable for definitive coverage measures such as skin grafting. After application of UBM, the patient's wound was given time to remodel into a viable, vascularized wound bed with healthy granulation tissue to permit secondary coverage with dermal regenerates, skin grafting and/or tissue

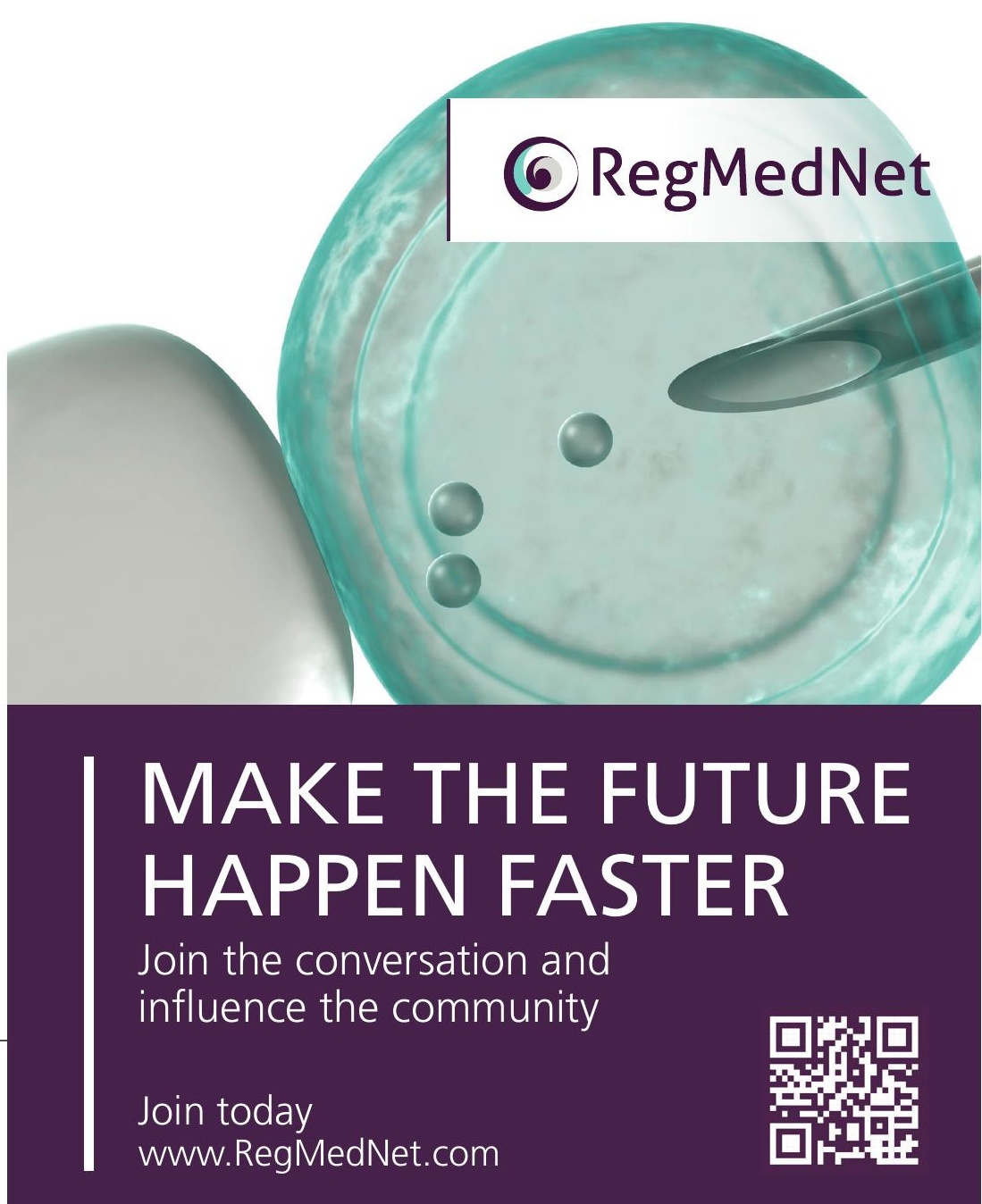


Table 1. Demographics, wound types and coverage outcomes/failures of urinary bladder matrix cases reviewed.

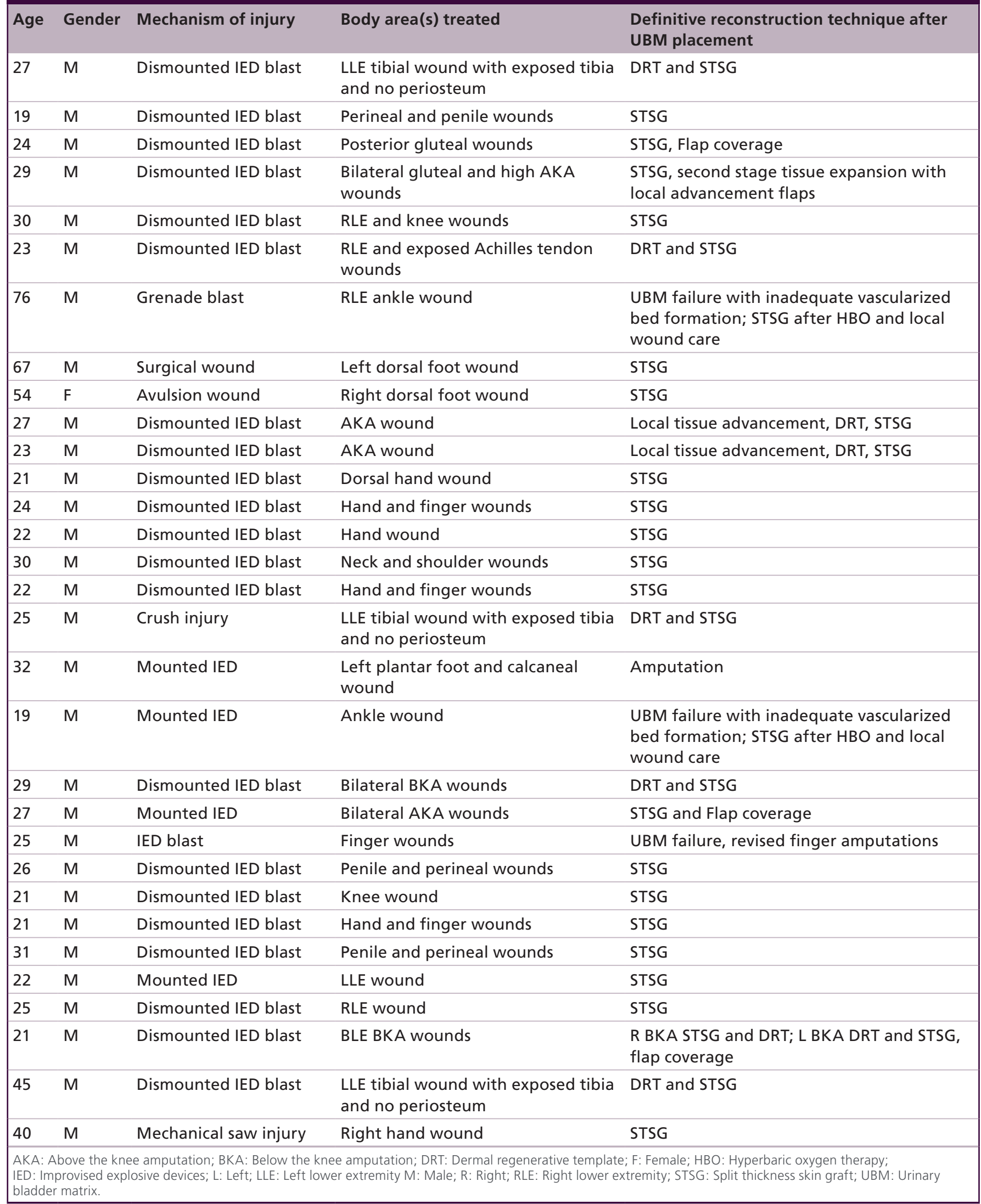


Table 1. Demographics, wound types and coverage outcomes/failures of urinary bladder matrix cases reviewed (cont.).

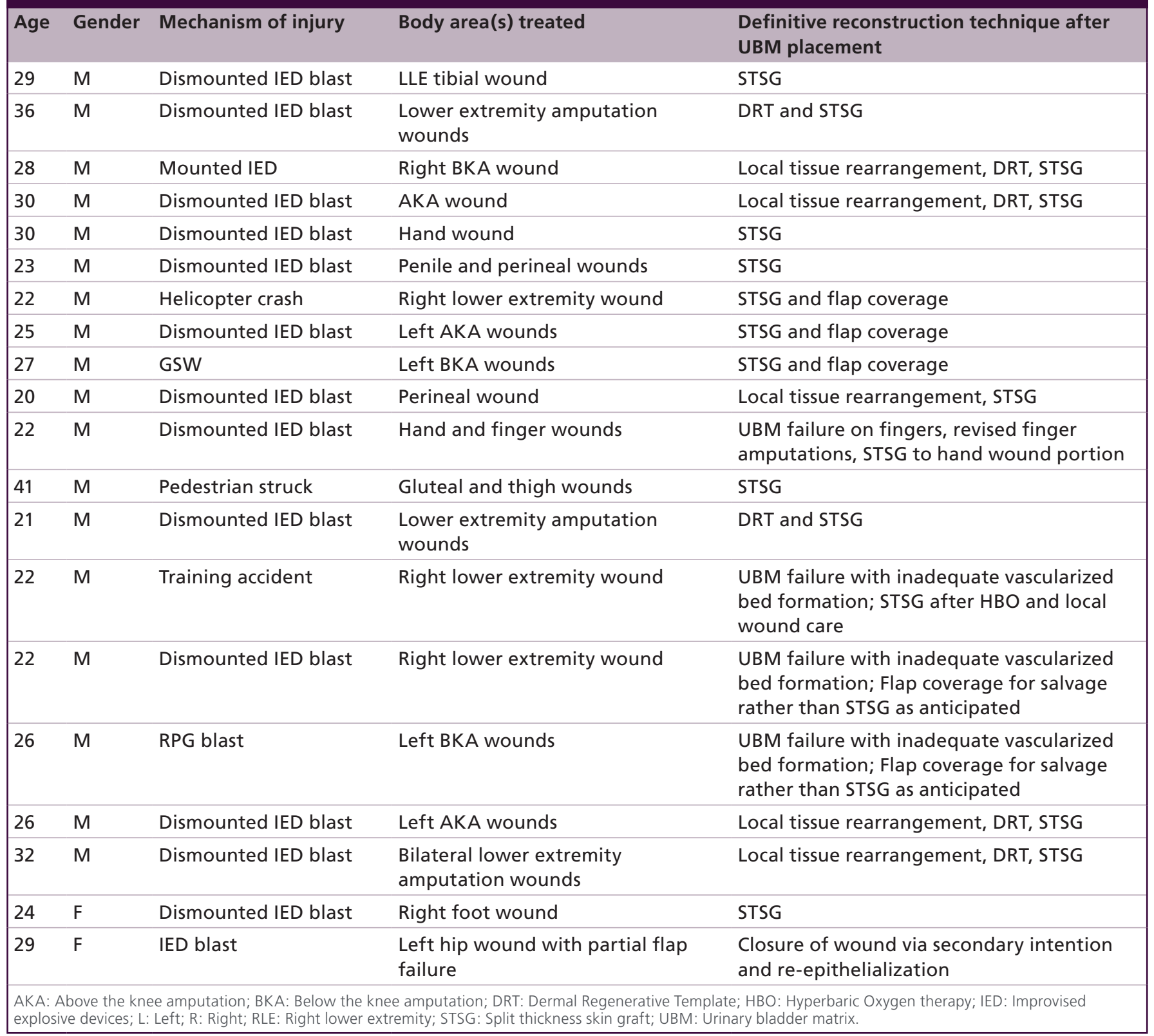

transfers (flaps). The patients were continuously monitored and their wounds were evaluated until definitive, stable coverage of the defect was successfully achieved.

\section{Results}

This clinical case series outlines 51 total cases as well as provides further details on four specific case examples in which UBM was utilized to treat various combat casualty wounds where prior attempts at facilitating wound healing had failed (Table 1). In general, UBM stimulated granulation tissue formation and/or directly aided in wound bed preparation by providing a neovascularized substrate to aid in re-epithelialization, secondary dermal regeneration template (DRT) placement, skin grafting and/or flap coverage. In the majority of cases (44/51 or $86 \%$ ), UBM successfully stimulated granulation tissue formation and/or directly aided in wound bed preparation to aid in re-epithelialization, secondary DRT placement, skin grafting and/or flap coverage. UBM failures occurred in $7 / 51$ cases $(14 \%)$ and were most commonly associated with material losses secondary to frictional forces/shearing, debridement of early remodeled tissue, superficial wound infections or inadequate neo- 


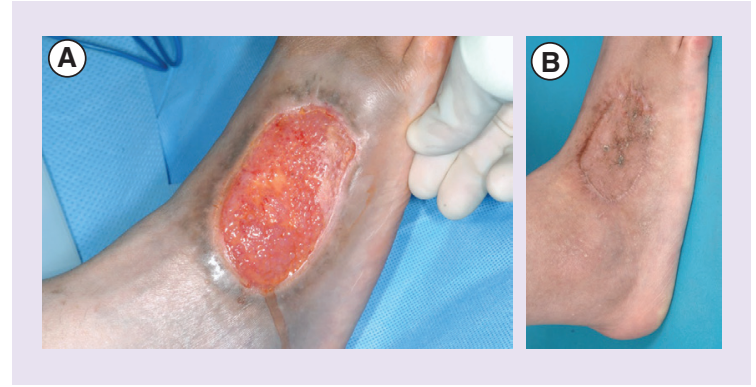

Figure 2. Right foot injury with original defect size of $6 \times 12 \mathrm{~cm}$ and having exposed extensor tendons after urinary bladder matrix incorporation. (A) Note regeneration of soft tissue and creeping edge of re-epithelialization along border of wound. (B) Right foot 2 years after complete re-epithelialization of the urinary bladder matrix. Patient has been able to ambulate, use footwear, and has not had breakdown of the regenerated dermal or epidermal skin areas of her reconstruction.

vascularization requiring more advanced wound care therapies measures (e.g., hyperbaric oxygen).

\section{Case examples illustrating results \& technique Case 1}

A 54-year-old female suffered an avulsion injury to the right dorsal foot which resulted in a full thickness $12 \times 6 \mathrm{~cm}$ defect containing exposed extensor tendons without paratenon to the second through fifth toes. The wound underwent serial irrigation and debridement procedures followed by placement of $200 \mathrm{mg}$ of UBM powder and a $7 \times 10 \mathrm{~cm}$ multilayer vacuum pressed UBM sheet over the defect. After 2 weeks, an additional 200 $\mathrm{mg}$ of UBM and another $7 \times 10 \mathrm{~cm}$ multilayer UBM sheet was placed in an effort to layer the defect with UBM until the wound and surrounding dorsal foot epidermis had a minimal step-off. The wound exhibited a viable soft tissue regenerate within 6 weeks following UBM placement (Figure 2A). This defect was then covered with a split thickness skin graft which has exhibited stable epidermal and dermal coverage without erosion over a 24-month period (Figure 2B).

\section{Case 2}

A 28-year-old US Service member (SM) suffered blast injuries resulting in trauma to multiple extremities including a right large soft tissue degloving injury and open elbow joint injury. Initial limb salvage reconstruction and coverage of the open elbow joint was attempted with a pedicle latissimus flap and DRT. However, the procedure was complicated by partial flap and DRT failure over the distal aspect of the elbow (Figure 3A). Exposure of devascularized tendon, especially over frequently used areas such as the elbow, is concerning as skin grafting to that area has high failure and ulceration rates due to shearing and insufficient vascularized tissue. Thus,
$100 \mathrm{mg}$ of UBM powder and a single $7 \times 10 \mathrm{~cm} \mathrm{UBM}$ sheet was utilized in an effort to stimulate formation of soft tissue over the exposed wound site (Figure 3B \& C). The wound and freshly placed UBM materials, prior incorporated DRT and remaining latissimus flap were then directly covered with over $800 \mathrm{~cm}^{2}$ of split thickness skin grafts (STSGs) (Figure 3D). Two weeks following the hybrid reconstruction, there was approximately $95 \%$ of STSG take with $100 \%$ viability of the STSG over the area in which UBM was placed. The hybrid salvage procedure provided stable, definitive soft tissue coverage without ulceration or wound complications throughout the 40-month follow-up period.

\section{Case 3}

A 27-year-old US SM suffered a blast which resulted in trauma to three extremities including a right below the knee amputation, a large soft tissue degloving and Gustilo-Anderson 3B injury of the left lower extremity (LLE) (Figure 4A), left open metacarpal fractures, as well as numerous facial fractures and perineal injuries. The patient was interested in limb salvage options for the lower extremity wound. After serial debridements, the LLE underwent reconstruction via the following hybrid reconstruction techniques. First, a left latissimus dorsi free flap was performed and was able to cover the proximal $2 / 3$ of the exposed bone. The distal $1 / 3$ of the wound and exposed bone was covered with a DRT that was placed over the tibia after drilling multiple cortical openings to aid in DRT incorporation. Unfortunately, the DRT failed leaving a significant portion of tibia bone devoid of periosteum exposed. UBM was then used as a salvage procedure; specifically, UBM powder and an overlying sheet of UBM were placed in the wound area and subsequently covered with NPWT. After three consecutive applications of the aforementioned technique, each spaced 3 days apart, a healthy granulated tissue regenerate completely covering the previous distal $1 / 3$ of exposed tibia was obtained (Figure 4B). Subsequently, $1008 \mathrm{~cm}^{2}$ of STSG was placed on the LLE - directly over the prior flap and UBM induced granulation tissue (Figure 4C). This hybrid reconstruction and salvage of the LLE has been stable (i.e., no significant wound healing issues or breakdown) for over 38 months and patient able to perform extreme athletic activities (patient is avid monoskier and competes at professional level).

\section{Case 4}

Following a dismounted blast, a 23-year-old US SM suffered significant lower extremity trauma to include a left below the knee amputation, and fractures of the right calcaneus, navicular, tibia and fibular with sig- 
nificant concomitant Gustilo-Anderson 3B soft tissue injuries to the proximal RLE. The reconstructive team performed a scapular free flap to the RLE for coverage of an exposed tibia fracture and placed two DRTs over the soft issue defect proximal to the flap (Figure 5A). However, on postoperative day seven, removal of the NPWT showed an $8 \times 2 \mathrm{~cm}$ area overlying the Achilles tendon where the DTR did not take. In an effort to salvage the failed DRT, two serial applications of UBM powder $(60 \mathrm{mg})$ and a $7 \times 10 \mathrm{~cm}$ lyophilized sheet of UBM were placed in the defect area 3 days apart (Figure 5B \& C). After 7 days, the previously exposed tendon developed a suitable layer of granulation tissue (Figure 5D) which permitted skin grafting for definitive coverage of the wound (Figure $5 \mathrm{E}$ ). The hybrid salvage reconstruction has been stable for over 42 months, without any significant postoperative soft tissue or wound breakdown and his external hardware removed after healing of his fracture.

\section{Discussion}

Postoperative management of complex open wounds is a challenging task. The patients within the current case series sustained significant traumatic and combat wounds resulting in volumetric soft tissue deficits, exposed joints, and stripped paratenon, periosteum and perineurium, which are nearly devoid of functional vasculature bed. The use of UBM as a supplement to traumatic and combat wounds where prior attempts at facilitating wound healing had failed was evaluated. Overall, UBM was found to facilitate constructive remodeling of the injury site into a healthy vascularized wound bed capable of supporting further advanced reconstruction efforts, such as definitive coverage with grafts or flaps [26-28]. The
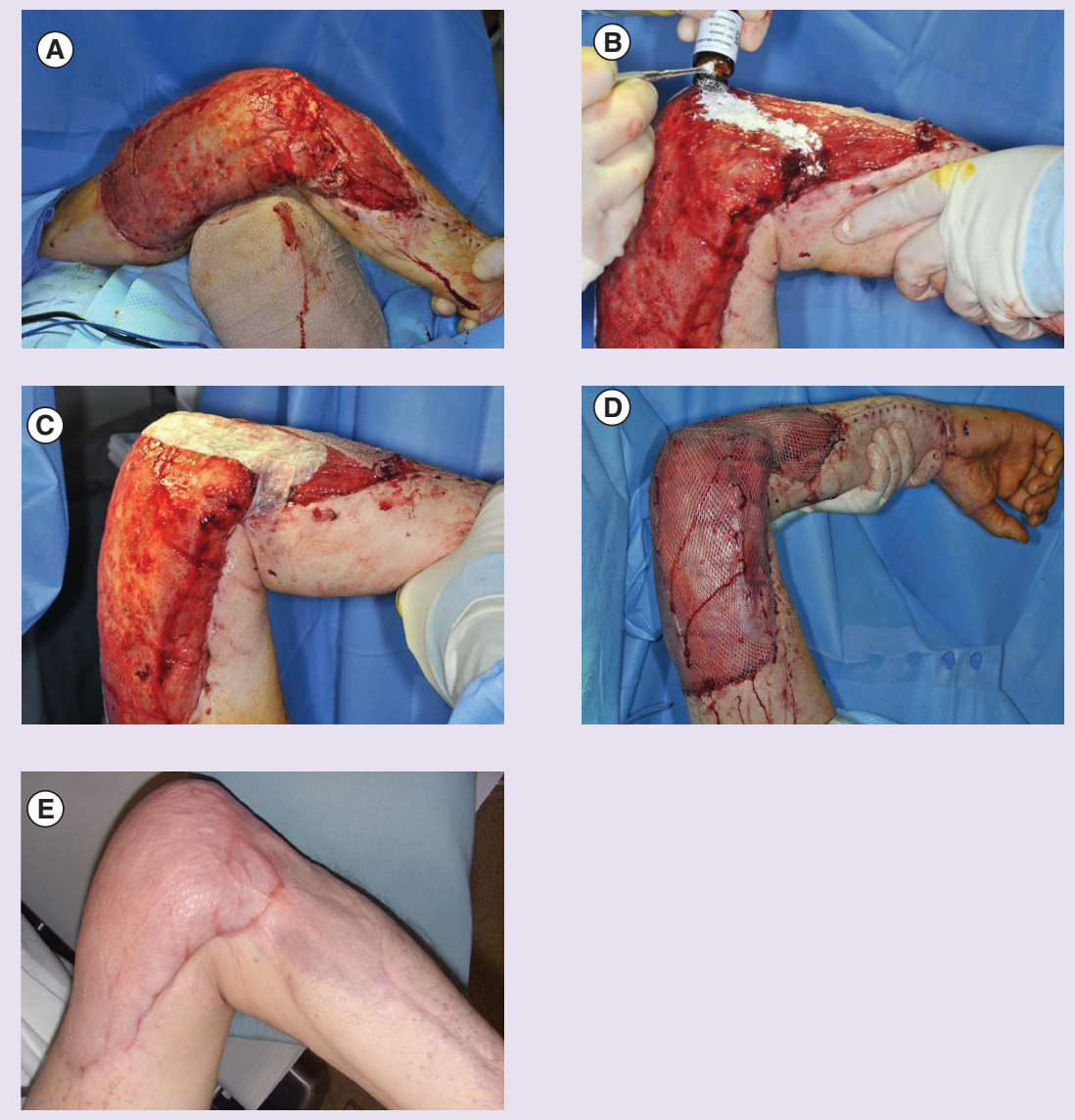

Figure 3. Case 2: mangled upper extremity. (A) RUE hybrid reconstruction case with latissimus flap and dermal regenerative template to exposed elbow defect. Note the failed flap and dermal regenerative template with exposed tendon devoid of paratenon over the elbow. (B) RUE hybrid salvage procedure with placement of urinary bladder matrix (UBM) powder over exposed tendon devoid of paratenon and the elbow wound where prior noted failed soft tissue reconstruction was apparent. (C) RUE hybrid salvage procedure with placement of UBM sheet covering prior UBM powder placement and prior to immediate skin grafting procedure. (D) RUE hybrid salvage procedure with placement of split thickness skin graft over the UBM material construct and final intraoperative appearance. (E) RUE 2 years post-UBM salvage procedure and split thickness skin graft reconstruction. 

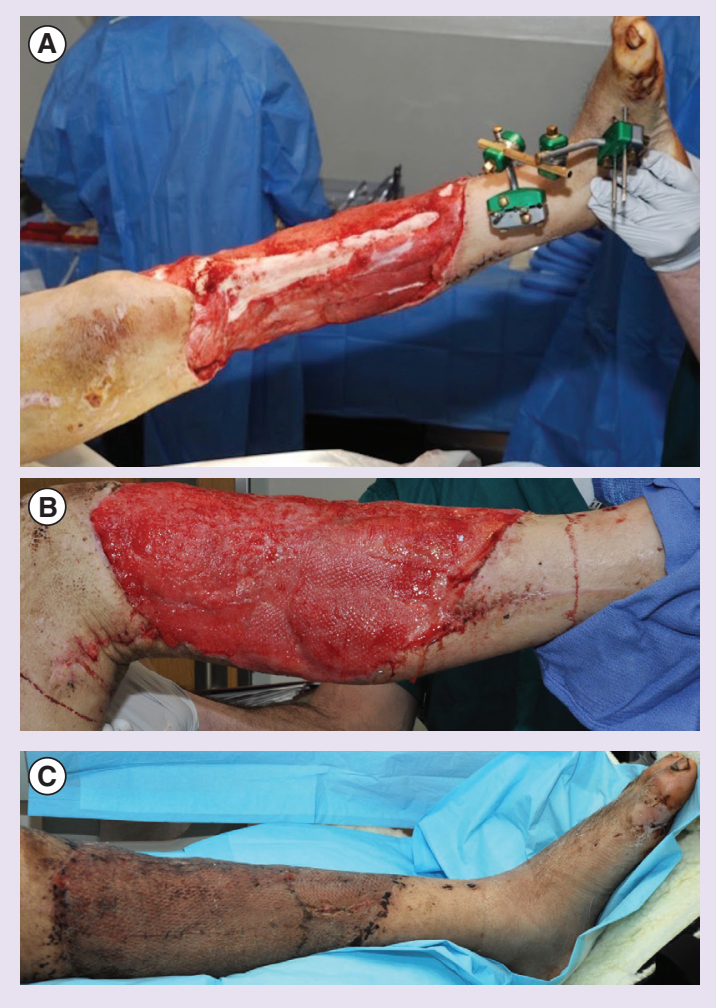

Figure 4. Case 5. (A) Left lower extremity (LLE) blastrelated trauma showing severe soft tissue degloving and grade $3 \mathrm{~B}$ exposed tibial fracture prior to limb salvage. Note significant portion of tibia devoid of periosteum. (B) LLE reconstruction after free latissimus flap placed over the proximal half and urinary bladder matrix regenerate over the distal half of prior exposed tibia. Flap covers superior $2 / 3$ of exposed bone and distal $1 / 3$ is soft tissue regenerate secondary to urinary bladder matrix scaffold placement showing well vascularized tissue bed establishment with coverage of exposed bone. The urinary bladder matrix aided in establishing vascularized bed favorable for skin grafting. (C) LLE reconstruction appearance 1 month after split thickness skin graft over hybrid reconstruction salvage and urinary bladder matrix regenerate.

average time of wound closure varied, which was dependent on a number of factors, including the magnitude and complexity of the wound and comorbid conditions.

Currently, there exist more than 60 commercially available biologic scaffold products, derived from a wide variety of tissues both allogeneic and xenogeneic in origin, and companies that continue to commercialize new materials. Thousands of patients have been treated with biologic scaffold materials. There are multiple clinical applications and many anatomical sites that benefit from treatment with biologic scaffolds. Biologic scaffolds have been shown to facilitate limited tissue remodeling in a number of tissues in both preclinical animal models and human clinical studies [26-39]. The host response to biologic scaffold has been extensively characterized. When appropriately prepared, biologic scaffolds are rapidly infiltrated by mononuclear cells, which mediate the degradation of the scaffold over the subsequent weeks to months. During this time, biologic scaffold materials orchestrate a complex temporospatial host response that includes modulation of the innate immune response, the recruitment, proliferation and differentiation of multipotent stem/progenitor cells, and ultimately the formation of site appropriate tissue.

Biologic scaffold materials have been shown to promote angiogenesis within weeks of being surgically placed at an injury site [34,35]. As the ECM is degraded by host cells, soluble factors such as vascular endothelial growth factor can be liberated from the scaffold which may aid in this process, as vascular endothelial growth factor is known to promote key components of angiogenesis and neovascularization [30-33]. Furthermore, the benefit of proangiogenic properties has also been linked to increases in graft and flap survival [35]. Many of the traumatic injuries presented herein had compromised vascular beds (i.e., tendon exposure lacking paratenon, bone devoid of periosteum), and thus benefited from the proangiogenic effects of UBM in the preparation of a healthy vascularized wound bed for eventual definitive tertiary reconstruction techniques.

As mentioned previously, infection rates in wounded military personnel can be significantly elevated due in large part to the mechanism of blast-related exposures. Blast injuries create diffuse areas of soft tissue destruction along with bacterial impregnation into wounds that directly correlate with increase rates of infection [36]. The large areas of uncovered defects in the soft tissue only serve to increase rates of infection. Flap infections are potentially devastating as they result in increased flap failure and further morbidity to the patient and contribute to limb loss or more proximal amputation revision rates [37]. Biologic scaffolds have been shown to possess antimicrobial properties. Following the direct inoculation of the pathogens Staphylococcus Aureus and Escherichia Coli, biologic scaffolds have been shown to inhibit their growth for 24 and 9 h, respectively [38]. Furthermore, biologic scaffolds have been shown to have significantly lower bacterial loads, decreased rates of antibiotic use and negative joint cultures compared with synthetic mesh following direct inoculation of $S$. Aureus after joint reconstruction in dogs [21]. These antimicrobial attributes of biologic scaffolds make them suitable materials for use prior to definitive reconstruction procedures, particularly in the military trauma patient.

There are several advantages to using UBM to treat acute and chronic wounds when compared to other therapies. Compared to traditional flap reconstruction, pilonidal disease treated with UBM resulted in shorter 

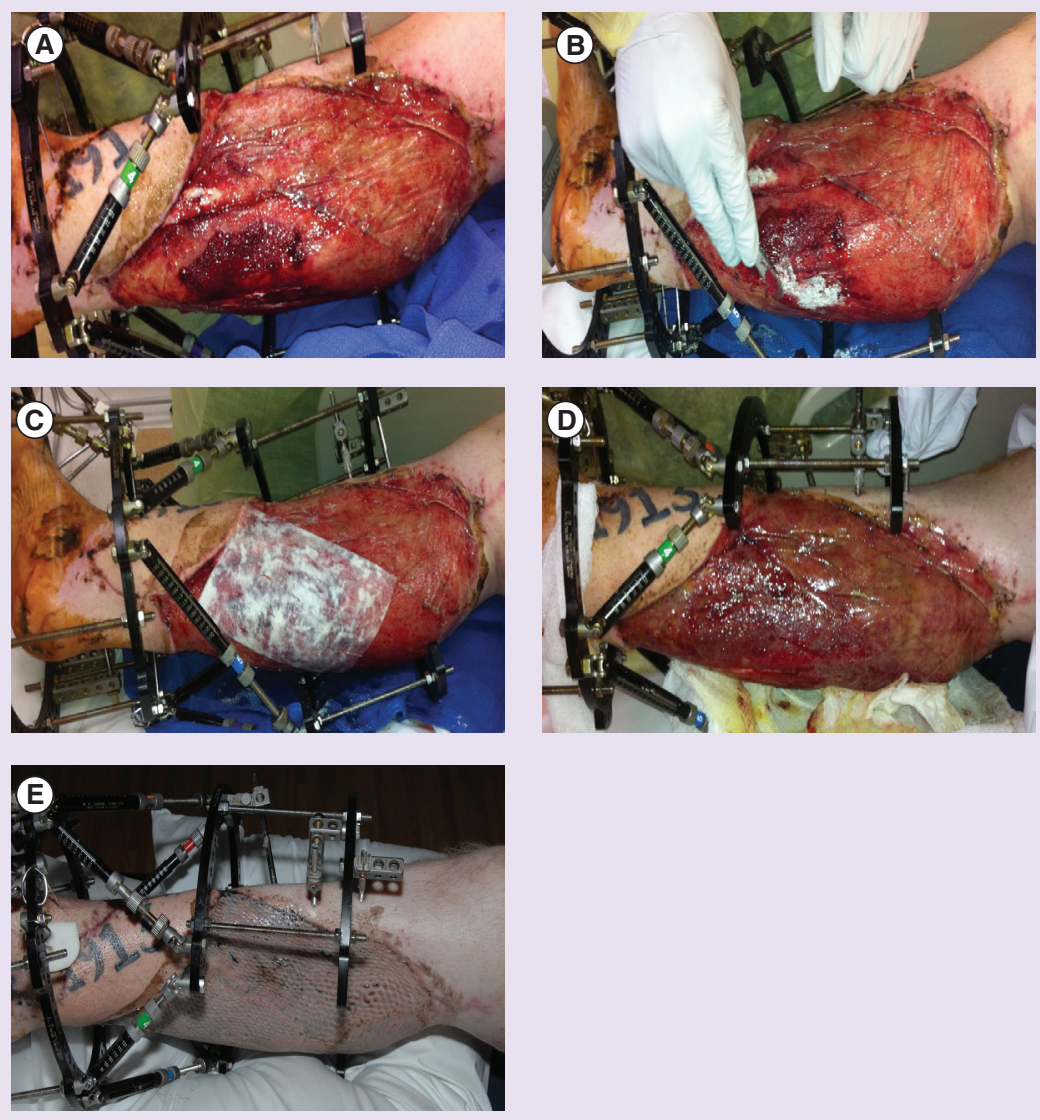

Figure 5. Case 4: mangled right lower extremity. (A) Right lower extremity (RLE) hybrid reconstruction illustrating scapular free flap for distal tibia fracture coverage (fracture stabilized by Taylor Spatial Frame) and dermal regenerative template coverage of remaining significant soft tissue defect. Note the exposed tendon where the dermal regenerative template failed to incorporate secondary to insufficient paratenon and vascularized tissue base. (B) RLE hybrid reconstruction salvage with placement of urinary bladder matrix (UBM) powder over exposed tendon. (C) RLE hybrid reconstruction salvage with placement of UBM sheet over the powder and prior exposed tendon. A total of two applications of UBM powder and sheets was performed 3 days apart with negative pressure therapy over the UBM constructs. (D) RLE hybrid reconstruction salvage after 7 days and two serial applications of UBM powder and sheets. Note the visible layer of vascularized granulation tissue base acceptable for skin grafting. (E) RLE salvage reconstruction illustrating complete take of split thickness skin graft and viable, durable coverage of prior exposed tendon. Patient RLE hybrid salvage was successful with no failures or soft tissue breakdown during his postoperative recovery and rehabilitation course.

duration of therapy, hospital stay and complication rate $[39,40]$. UBM has also been used to successfully salvage failed regional flaps after oncologic resection [41]. However, to date, there is a paucity of evidence to demonstrate whether UBM performs better than traditional dressings or other biologic adjunctive therapies given the inherent confounding factors associated with comparing treatment modalities between patients.

Despite the improved outcomes observed with the use of UBM for reconstructive purposes, multiple limitations to our case series exist. All of the definitive reconstructive treatments took place at one institution, but prior to arrival each wounded military member received varying numbers of surgical procedures, antibiotics and debridements from multiple providers. The primary mechanism of injury was improvised explosive devices blasts, but the injury pattern and extent of tissue loss was highly variable and unique to each individual.

Utilizing the beneficial remodeling aspects of an ECM scaffold material, we have effectively converted full thickness soft tissue wounds into partial thickness wounds with re-establishment and restoration of subepidermal and dermal-replacement structures. These restored subepidermal soft tissue and elements have permitted the application of skin grafts as well as regenerative cell-based therapies including spray skin technologies to allow for re-epithelialization of these partial thickness wounds into stable, multilayered skin regenerates that have proven to be durable and viable over time in various clinical applications [42]. 


\section{Future perspective}

The field of Regenerative Medicine has developed strategies that facilitate the restoration of form and function to damaged tissues. As discussed herein, the use of UBM, an ECM-derived biologic scaffold, can be used to support site appropriate constructive tissue remodeling of traumatic and combat related extremity injuries. While these results are promising, additional prospective, randomized and controlled studies are needed to build upon this success. Furthermore, as our understanding of the mechanisms which underlie biologic scaffold mediated constructive remodeling improves and molecular targets are identified, rationally designed, next generation materials can be designed to facilitate improved tissue form/function and patient quality of life.

\section{Disclaimer}

The views expressed in this presentation are those of the authors and do not necessarily reflect the official policy or position of the Department of the Army, Department of Defense, nor the US Government.

Financial \& competing interests disclosure

The authors have no relevant affiliations or financial involvement with any organization or entity with a financial interest in or financial conflict with the subject matter or materials discussed in the manuscript. This includes employment, consultancies, honoraria, stock ownership or options, expert testimony, grants or patents received or pending, or royalties.

No writing assistance was utilized in the production of this manuscript.

\section{Executive summary}

- War-related trauma can result in complex soft tissue wounds requiring regenerative medicine modalities and appropriate wound care measures. Traumatic wounds may be complicated by exposure of vital structures devoid of well vascularized adjacent tissue.

- Regenerative medicine therapies along with traditional soft tissue coverage techniques play a role in treating complex wounds.

- Urinary bladder matrix (UBM) is an acellular, noncross-linked, resorbable, biologically-derived extracellular matrix (ECM) scaffold that can aid in establishing a neovascularized wound bed in certain wounds.

- The use of UBM, in combination with other reconstructive techniques, has been demonstrated to be a successful adjunct in the reconstructive algorithm for complex traumatic wound reconstruction and limb salvage procedures.

Aims

- Due to the destructive nature of low- and high-energy wounding mechanisms, massive soft tissue defects are encountered at ever increasing rates. Treatment of wounds secondary to war-related trauma requires extensive surgical care algorithms with complex salvage techniques to accomplish stable, definitive wound coverage. Commonly, exposed at-risk structures, such as tendons, nerves, blood vessels and bone, require the creation of durable, vascularized coverage to facilitate healing. The goal is to provide a viable wound environment to achieve successful wound control and/or coverage in order to allow the patient to begin rehabilitation. This clinical case series reports on the regenerative potential of urinary bladder matrix (UBM) scaffolds in the reconstruction of traumatic combat wounds.

\section{Materials \& methods}

- A retrospective review of combat casualty cases employing UBM for the treatment of traumatic wounds was performed. All cases reviewed were performed at Walter Reed National Military Medical Center, Bethesda, MD, USA between 2010 and 2014. UBM is an acellular, noncross-linked, resorbable ECM biologic scaffold, available in powder and sheet forms. The data collected and analyzed during the retrospective review included: patient demographics, injury sites, number and type of UBM treatments, complications and overall tissue remodeling outcome.

\section{Results}

- This clinical case series details a number of examples in which UBM was utilized to treat various combat casualty wounds. UBM was found to facilitate granulation tissue formation, a neovascularized wound bed, and directly aided in wound bed preparation for re-epithelialization, secondary dermal regeneration template placement, skin grafting and/or flap coverage.

Conclusion

- The use of regenerative medicine modalities such as UBM derived ECMs in the management of combat casualties continues to expand. The incorporation of UBM derived ECMs in surgical treatment algorithms can facilitate achieving stable wounds, which leads to improve outcomes. The current report illustrates the ability of UBM to facilitate definitive soft tissue reconstruction via establishment of neovascularized wound beds acceptable for second stage wound and skin coverage options within traumatic and combat-related wounds. 


\section{References}

Papers of special note have been highlighted as:

- of interest

1 Eastridge BJ, Mabry RL, Seguin P et al. Death on the battlefield (2001-2011), implications for the future of combat casualty care. J. Trauma Acute Care Surg. 73(6 Suppl. 5), S431-S437 (2012).

2 Kotwal RS, Montgomery HR, Kotwal BM et al. Eliminating preventable death on the battlefield. Arch. Surg. 146(12), 1350-1358 (2011).

3 Gillern SM, Sheppard FR, Evans KN et al. Incidence of pulmonary embolus in combat casualties with extremity amputations and fractures. J. Trauma 71(3), 607-612 (2011).

4 Borgman MA, Spinella PC, Perkins JG et al. The ratio of blood products transfused affects mortality in patients receiving massive transfusions at a combat support hospital. J. Trauma 63(4), 805-813 (2007).

5 CRASH-2 trial collaborators, Shakur H, Roberts I, Bautista $\mathrm{R}$ et al. Effects of tranexamic acid on death, vascular occlusive events, and blood transfusion in trauma patients with significant haemorrhage (CRASH-2), a randomised, placebo-controlled trial. Lancet 376(9734), 23-32 (2010).

6 Morrison JJ, Dubose JJ, Rasmussen TE, Midwinter MJ. Military application of tranexamic acid in trauma emergency resuscitation (MATTERs) study. Arch. Surg. 147(2), 113-119 (2012).

$7 \quad$ Krueger CA, Wenke JC, Ficke JR. Ten years at war: comprehensive analysis of amputation trends. J. Trauma Acute Care Surg. 73(6 Suppl. 5), S438-S444 (2012).

8 Morrison JJ, Hunt N, Midwinter M, Jansen J. Associated injuries in casualties with traumatic lower extremity amputations caused by improvised explosive devices. $B r$. J. Surg. 99(3), 362-366 (2012).

9 Doucet JJ, Galarneau MR, Potenza BM et al. Combat versus civilian open tibia fractures: the effect of blast mechanism on limb salvage. J. Trauma 70(5), 1241-1247 (2011).

10 Fleming ME, Bharmal H, Valerio I. Regenerative medicine applications in combat casualty care. Regen. Med. 9(2), 179-190 (2014).

- Describes the use of novel hybrid reconstruction techniques, which combine traditional and regenerative medicine approaches, as demonstrates their effectiveness in managing complex wounds sustained by service members.

11 Mamczak CN, Elster EA. Complex dismounted IED blast injuries: the initial management of bilateral lower extremity amputations with and without pelvic and perineal involvement. J. Surg. Orthop. Adv. 21(1), 8-14 (2012).

12 Ramasamy A, Harrisson SE, Clasper JC, Stewart MP. Injuries from roadside improvised explosive devices. J. Trauma 65(4), 910-914 (2008).

13 Badylak SF. The extracellular matrix as a scaffold for tissue reconstruction. Semin. Cell Dev. Biol. 13(5), 377-383 (2002).

14 Badylak SF, Freytes DO, Gilbert TW. Extracellular matrix as a biological scaffold material: structure and function. Acta Biomater. 5(1), 1-13 (2009).
15 Cornwell KG, Landsman A, James KS. Extracellular matrix biomaterials for soft tissue repair. Clin. Podiatr. Med. Surg. 25(4), 507-523 (2009).

16 Badylak SF. The extracellular matrix as a biologic scaffold material. Biomaterials 28(25), 3587-3593 (2007).

17 Moiemen NS, Vlachou E, Staiano JJ, Thawy Y, Frame JD. Reconstructive surgery with Integra dermal regeneration template: histologic study, clinical evaluation, and current practice. Plast. Reconstr. Surg. 117(7 Suppl.), S160-S174 (2006).

18 Fitton AR, Drew P, Dickson WA. The use of bilaminate artificial skin substitute (Integra) in acute resurfacing of burns: an early experience. Br. J. Plast. Surg. 54(3), 208-212 (2001).

19 Beattie AJ, Gilbert TW, Guyot JP, Yates AJ, Badylak SF. Chemoattraction of progenitor cells by remodeling extracellular matrix scaffolds. Tissue Eng. A 15(5), 119-125 (2009).

20 Turner NJ, Badylak SF. The use of biologic scaffolds in the treatment of chronic nonhealing wounds. Adv. Wound Care doi:10.1089/wound.2014.0604 (2014) (Epub ahead of print).

21 Brennan EP, Reing J, Chew D, Myers-Irvin J M, Young EJ, Badylak SF. Antibacterial activity within degradation products of biological scaffolds composed of extracellular matrix. Tissue Eng. 12(10), 2949-2955 (2006).

22 Gilbert TW, Wognum S, Joyce EM, Freytes DO, Sacks MS, Badylak SF. Collagen fiber alignment and biaxial mechanical behavior of porcine urinary bladder derived extracellular matrix. Biomaterials 29(36), 4775-4782 (2008).

23 Hodde J. Naturally occurring scaffolds for soft tissue repair and regeneration. Tissue Eng. 8(2), 295-308 (2002).

24 Reing JE, Zhang L, Myers-Irvin J et al. Degradation products of extracellular matrix affect cell migration and proliferation. Tissue Eng. A 15(3), 605-614 (2009).

25 Badylak SF. Xenogeneic extracellular matrix as a scaffold for tissue reconstruction. Transpl. Immunol. 12(3), 367-377 (2004).

26 Israeli R, Funk S, Reaven NL. Comparative analysis of 18-month outcomes and costs of breast reconstruction flap procedures. Plast. Reconstr. Surg. 133(3), 471-479 (2014).

27 Badylak SF. Decellularized allogeneic and xenogeneic tissue as a bioscaffold for regenerative medicine: factors that influence the host response. Ann. Biomed. Eng. 42(7), 1517-1527 (2014).

28 Lou RB, Hickerson WL. The use of skin substitutes in hand burns. Hand Clin. 25(4), 497-509 (2009).

29 Turner NJ, Yates AJ Jr, Weber DJ et al. Xenogeneic extracellular matrix as an inductive scaffold for regeneration of a functioning musculotendinous junction. Tissue Eng. A 16(11), 3309-3317 (2010).

30 Greaves NS, Iqbal SA, Baqueid M, Bayat A. The role of skin substitues in the management of chronic cutaneous wounds. Wound Repair Regen. 21(2), 194-210 (2013).

31 Brown BN, Londono R, Tottey S et al. Macrophage phenotype as a predictor of constructive remodeling following the implantation of biologically derived surgical mesh materials. Acta Biomater. 8(3), 978-987 (2012). Bellon G, Martiny L, Robinet A. Matrix metalloproteinases and matrikines in angiogenesis. Crit. Rev. Oncol. Hematol. 49(3), 203-220 (2004). 
33 Lee S, Jilani SM, Nikolova GV, Carpizo D, Iruela-Arispe ML. Processing of VEGF-A by matrix metalloproteinases regulates bioavailability and vascular patterning in tumors. J. Cell Biol. 169(4), 681-691 (2005).

34 Monteiro GA, Rodriguez NL, Delossantos AI, Wagner CT. Short-term in vivo biological and mechanical remodeling of porcine acellular dermal matrices. J. Tissue Eng. doi:10.1177/2041731413490182 (2013) (Epub ahead of print).

35 Garcia O Jr, Scott JR. Analysis of acellular dermal matrix integration and revascularization following tissue expander breast reconstruction in a clinically relevant large-animal model. Plast. Reconstr. Surg. 131(5), e741-e751 (2013).

36 Yan H, Black D, Jones NI et al. Integra acellular collagen as a vascular carrier for skin flap prefabrication in rats. Ann. Plast. Surg. 67(3), 299-302 (2011).

37 Mody RM, Zapor M, Hartzell JD et al. Infectious complications of damage control orthopedics in war trauma. J. Trauma 67(4), 758-761 (2009).

38 Rehim SA, Singhal M, Chung KC. Dermal skin substitutes for upper limb reconstruction: current status, indications, and contraindications. Hand Clin. 30(2), 239-252 (2014).
39 Sasse KC, Brandt J, Lim DC, Ackerman E. Accelerated healing of complex open pilonidal wounds using MatriStem extracellular matrix xenograft: nine cases. J. Surg. Case Rep. doi:10.1093/jscr/rjt025 (2013) (Epub ahead of print).

- Describes the use of a biologic scaffold material for the treatment of complex open pilonidal wounds which resulted in favorable wound healing.

40 McCallum IJ, King PM, Bruce J. Healing by primary closure versus open healing after surgery for pilonidal sinus: systematic review and meta-analysis. BMJ 336(7649), 868-871 (2008).

41 Kruper GJ, Vandegriend ZP, Lin HS, Zuliani GF. Salvage of failed local and regional flaps with porcine urinary bladder extracellular matrix aided tissue regeneration. Case Rep. Otolaryngol. doi:10.1155/2013/917183 (2013) (Epub ahead of print).

- Describes the use of a biologic scaffold material to salvage flaps with extensive wound breakdown.

42 Tenenhaus M, Rennekampff HO. Surgical advances in burn and reconstructive plastic surgery: new and emerging technologies. Clin. Plast. Surg. 39(4), 435-443 (2012). 